\title{
Design of Arbitrarily Shaped Planar Microstrip Antenna Arrays with Improved Efficiency
}

\author{
Sheng Ye, ${ }^{1}$ Xianling Liang, ${ }^{1}$ Wenzhi Wang, ${ }^{1}$ Junping Geng, ${ }^{1}$ Ronghong Jin, \\ Y. Jay Guo, ${ }^{2}$ and Trevor S. Bird ${ }^{2}$ \\ ${ }^{1}$ Department of Electronic Engineering, Shanghai Jiao Tong University, Shanghai 200030, China \\ ${ }^{2}$ Commonwealth Scientific and Industrial Research Organisation (CSIRO), Computational Informatics, P.O. Box 76, \\ Epping, NSW 1710, Australia \\ Correspondence should be addressed to Sheng Ye; zqntoys@sjtu.edu.cn
}

Received 24 March 2013; Revised 28 November 2013; Accepted 28 November 2013

Academic Editor: Bal Singh Virdee

Copyright (C) 2013 Sheng Ye et al. This is an open access article distributed under the Creative Commons Attribution License, which permits unrestricted use, distribution, and reproduction in any medium, provided the original work is properly cited.

\begin{abstract}
A design technique is described for an arbitrarily shaped planar microstrip antenna array with improved radiation efficiency. In order to fully utilize the limited antenna aperture, several basic modules are proposed from which we construct the array. A consideration of the aperture shape shows that with several practical examples a proper combination of these basic modules not only allows the convenient design of arbitrarily-shaped microstrip array, but also helps to improve the aperture radiation efficiency. To confirm the feasibility of the approach, a circular array with 256 elements was constructed and fabricated. Both computed and measured aperture radiation results are compared and these demonstrate that the design technique is effective for arbitrarily-shaped planar microstrip arrays.
\end{abstract}

\section{Introduction}

With the rapid deployment of wireless communications systems during recent years, it is becoming imperative for the radiofrequency subsystem to be multifunctional as well as have smaller size. This is especially true for satellite communications applications where weight and functionality are at a premium. As one of main parts in the wireless system, the antenna is required to perform at a high performance level, whilst maintaining conformability with the remainder of the system. Accordingly, making full use of the available system space is very important in future antenna design for on-board space communications due to space limitations.

Traditional parabolic or reflector antennas have a high radiation efficiency, and they have been employed successfully in current satellite communication systems $[1,2]$. However, they have a large profile and high volume due to their support structures. To utilize the system space more effectively, a planar array antenna with a low profile has significant potential for space saving: such antennas include the radial line slot antenna (RLSA) array, the waveguide slot antenna array [3-8], and planar microstrip antenna array [9]. However, the RLSA is commonly suitable for a circular space design, where the waveguide slot array antenna needs an additional power divider, which may not be suitable for the arbitrarily shaped array designs. By contrast, the planar microstrip antenna array is more adaptable and more easily made conformal with the system. For example, several special shaped microstrip arrays have been proposed and designed to be conformal in [10-14]. A circular array was designed in [10] for mobile TV reception applications, where three kinds of collinear subarrays are specially designed to be compatible with the curved edge, and an extra feed network is added to combine these subarrays together to provide a peak antenna gain of $27.4 \mathrm{dBi}$. A commercial product for mobile satellite communication in Ku-band, described in [13], adopts flat antenna boards to chamfer shapes so that the antenna can rotate mechanically in elevation without interfering with any 


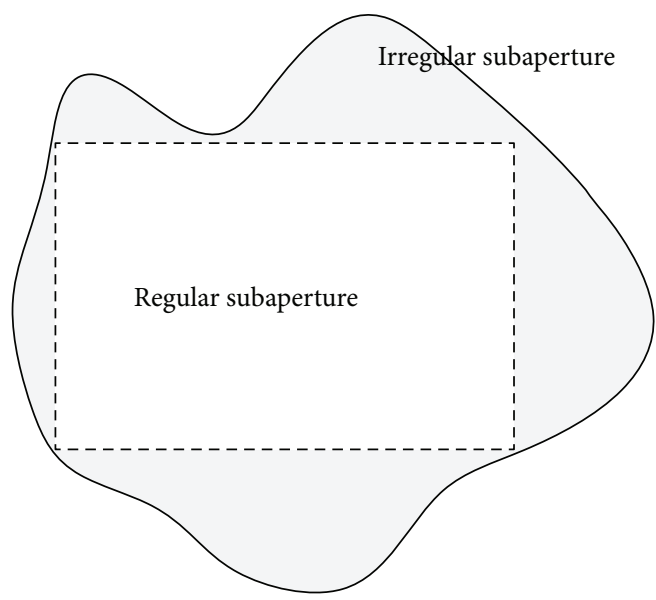

FIGURE 1: Layout of an arbitrarily shaped array.

radome of related structure. When combined together, the three panels produce a receive gain of $29.5 \mathrm{dBi}$. A hexagonshaped 12-element array designed for dual polarizations was reported in [12], to have a gain of $<17.5 \mathrm{dBi}$ at a frequency of $15.4 \mathrm{GHz}$. In [14], a symmetrical feed network composed of multiple T-junctions was specially designed for a $3 \times 2$ element array to achieve a gain of $17 \mathrm{dBi}$. All these antenna design examples were based on the available system space, but they exhibit no universality in either irregularity in the geometry or employ complicated geometrical shapes. Therefore, it is useful to investigate an arbitrarily shaped antenna array for better utilizing the available space. In [15], the authors gave a feed network layout of an $8 \times 8$-element array; the array is divided into 32 2-element subarrays, which are combined by a parallel and serial technique. A curved microstrip line and 1 to 3 power dividers make this different from reference [9-14]. Nevertheless the approach cannot be used to design arbitrarily shaped arrays as the feed network would be very complicated and the loss therein would be significant. Another disadvantage of the available design methods is that they all employ a single method to design the whole array, which may contain both regular and irregular subapertures, as shown in Figure 1. The traditional symmetry method is preferred for a regular subaperture because the symmetry network is beneficial in cancelling out spurious radiation. However, a regular symmetrical network cannot be applied to an irregular subaperture design due to the constancy of its layout.

In this paper, we present a design technique for an arbitrarily shaped antenna array. The array itself has a fixed beam that is scanned mechanically. In contrast with existing design methods, the regular and the irregular subapertures are treated differently with alternative design methods in order to achieve overall maximum gain. Moreover, small subarrays are employed in the construction of the irregular subaperture so that depending on the application it can be integrated with the regular subaperture. The design technique is easy to be realized with planar circuit fabrication; hence it is applicable to most wireless communications systems.
The paper is organized as follows. Section 2 describes an arbitrarily shaped array design approach, where several basic modules are introduced. A design guideline is given in Section 3 for the combination of the modules. In Section 4 a circular array is constructed and fabricated using the described method. Measured results are then reported and discussed to assess the theoretical design. Our conclusion is given in the final Section.

\section{Arbitrarily Shaped Array Design Principle}

An arbitrarily shaped array can be divided into regular and irregular subapertures as shown in Figure 1. For the regular subaperture, the number of elements is chosen to be $2^{N}(N=1,2,3 \ldots)$, which can be designed by conventional symmetrical methods. For example, in [9], a 4-element array was first designed as a sub-array and then a 16-element array was constructed by duplicating the sub-array twice with an appropriate feed network. An identical strategy can be employed to design a larger $2^{N}$-element array. On the other hand, for an array in their regular subaperture, the antenna structure and the number of elements depend on the aperture size and shape. In common with symmetrical arrays, an irregular subaperture has two major attributes: element distribution and a corresponding feed network.

Consider now an array element layout for the shape in Figure 1. Assume that their regular subaperture contains $M$ elements. Figure 2 shows several basic modules for one, two, and three elements. For a single element, there is only one case, denoted by $M_{1}$ (see Figure 2(a)). For two elements, two cases are possible based on the parallel and serial feeds, respectively, denoted by $M_{2-1}$ and $M_{2-2}$, as shown in Figures 2(b) and 2(c). For three elements, there are two kinds of distributions: one is linear and the other is triangular. Moreover, each distribution has four structures of feed network, as shown in Figure 2(d) to 2(k), which are indicated by $M_{3-1}$ to $M_{3-8}$.

The next module in the series, $M_{4}$, can be expressed as $M_{4}=M_{1}+M_{3}$ or $M_{4}=M_{2}+M_{2}$; hence, it is not difficult to construct $M_{4}$ using the previously mentioned modules $\mathrm{M}_{1}$, $\mathrm{M}_{2}$, and $\mathrm{M}_{3}$. Using the same strategy, $M_{N}$ can be expanded to $M_{N}=M_{(N-X)}+M_{X}(N>X)$.

The irregular subaperture of Figure 1 can also be formed from a combination of the basic modules shown in Figure 2 as will be described in the next Section.

\section{Design of Feed Network}

3.1. Typical Structures. In order to suggest a general approach, we describe firstly subapertures with notched and concave boundaries. Figures 3 and 4 show notched concave rectangular apertures, respectively. Both spaces capable of 19 elements, where the 3 elements (the irregular sub-aperture containing the missing elements) are located next to a $4 \times 4$-element square array (the regular sub-aperture). Each structure has two cases: a vertical and a horizontal polarization feed network. Here, we regard a $2 \times 2$-element array as the basic unit, and the conventional method of feeding the basic 


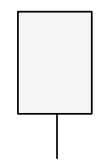

(a)

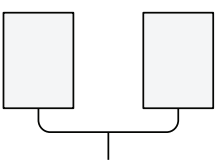

(b)

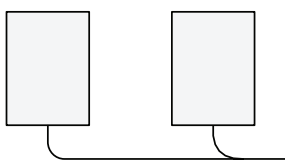

(c)

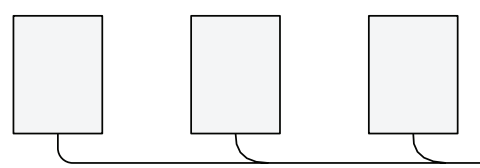

(d)

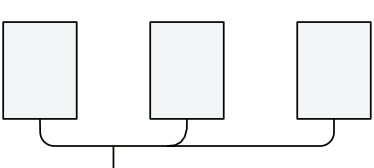

(e)

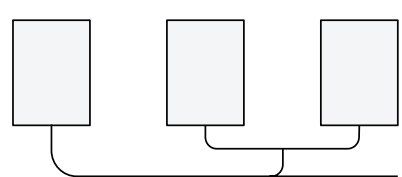

(f)

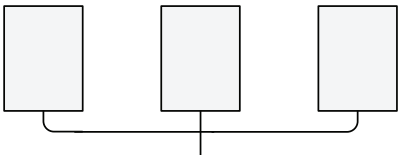

(g)

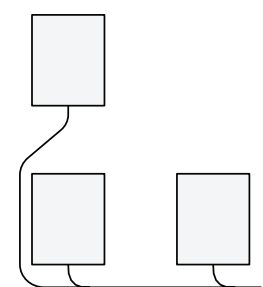

(h)

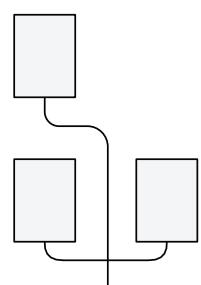

(i)

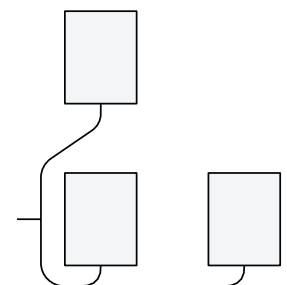

(j)

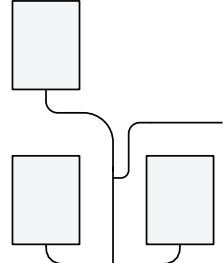

(k)

Figure 2: Configuration of the basic array element modules (a) $M_{1}$ (b) $M_{2-1}$ (c) $M_{2-2}$ (d) $M_{3-1}$ (e) $M_{3-2}$ (f) $M_{3-3}$ (g) $M_{3-4}$ (h) $M_{3-5}$ (i) $M_{3-6}$ (j) $M_{3-7}(\mathrm{k}) M_{3-8}$.

unit is simply using the serial and parallel techniques, in which multiple T-shaped power dividers are used. For the irregular subaperture, the three special elements (denoted by the dotted line) are commonly neglected in conventional design.

To improve the efficiency of the antenna aperture, three special elements designs need to be considered. Figures 3 and 4 show the corresponding two designs, namely, irregular design 1 and irregular design 2. Design 1 confines the feed network inside the area between regular and irregular subapertures, whereas in design 2 , it further divides the irregular subaperture into several smaller modules. The main differences between the two designs are the topology of the feed lines and the positions of feed junctions.

3.2. Simulated Results and Discussion. The array element designs were carried out with CST microwave studio (MWS). They were all modeled in the size indicated in Figures 3 and 4. Power is side fed through a waveguide port input. The adjustment of the microstrip network is now described. Firstly, all elements are tuned to have nearly uniform excitation. Secondly, the current travelling to the irregular subaperture is slightly reduced or increased until the gain of the complete array reaches its maximum value. During this process, the elements and the feed lines are matched throughout to maintain a reflection coefficient less than $-15 \mathrm{~dB}$ as shown in Figure 5. For the notched array with vertical polarization, array 2 exhibits a better return loss and a higher gain than array 1 . The feeding paths to module $M_{3}$ have almost the same length in both configurations; hence, it was concluded that in such cases the smaller module would be preferred. In both designs, the feed line used to connect the regular and irregular subapertures inevitably causes mutual coupling with the adjacent elements, and, further, the coupling effect is more severe as the currents become stronger. It is noted that the coupling will cancel out part of original currents on the element and cause the aperture efficiency to decrease. For array 1, unfortunately, the feed line from A1 to $M_{3}$ couples energy to adjacent elements along its path. Meanwhile, one section of the feed line carries a strong current; hence, the coupling is severe. By contrast, in array $2, M_{3}$ is divided into small modules so that the feed line current to each module is reduced. Therefore, the feed line will have coupling with the elements only when it is in the regular subaperture. Hence, small modules help reduce coupling effects where high aperture efficiency is required, as shown in Table 1 . The result for horizontal polarization is similar. Smaller modules in array 4 give a better performance than in array 3 . It should be pointed out that in array $4 M_{1}$ is not fed from the available junctions, but from newly created ones (B1, B2, and B3) in the feed lines, as illustrated in Figure 3(b). This is done to prevent changes at the higher level junctions in the basic unit so as to reduce the coupling effects.

For the concave array with vertical polarization, if $M_{3}$ is divided into the smaller modules, the feed network may be too complicated; hence the two designs differ only in feeding positions. Compared with array 5, array 6 has a little low aperture efficiency, as seen in Table 1 . It is caused by three junctions in array 6 , denoted by $\mathrm{C} 1, \mathrm{C} 2$, and $\mathrm{C} 3$, where the power ratios are $7: 4,2: 5$, and $1: 1: 3$, respectively, according to the uniform distribution. It is difficult to realize these power ratios accurately using the microstrip line, and impedance matching will not be properly achieved with the result that the loss with feed line increases and aperture efficiency decreases. Instead, the elements in array 


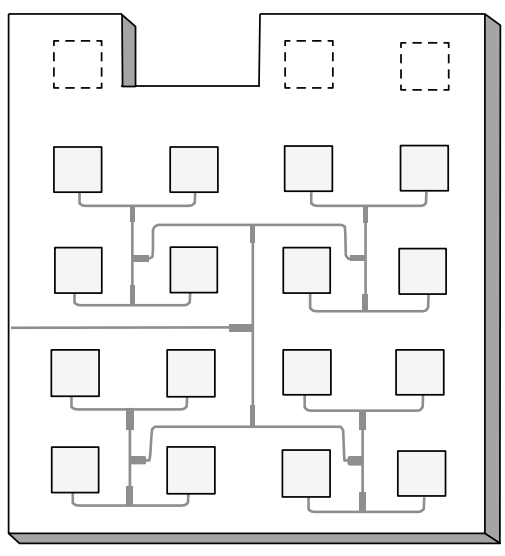

Regular design

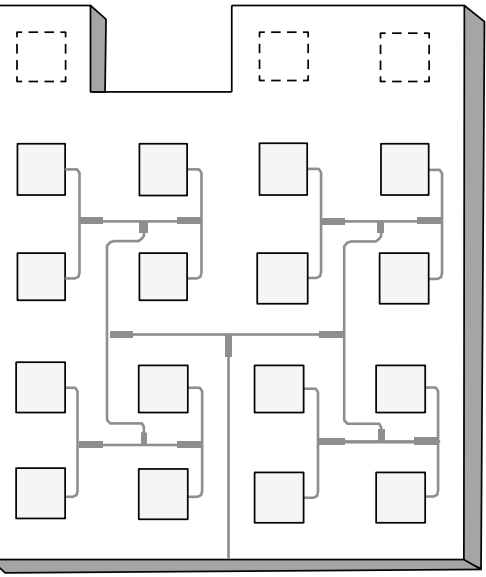

Regular design

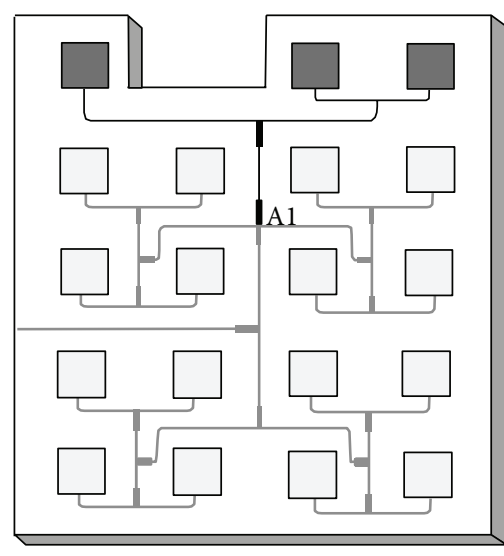

Irregular design 1 (array 1)

(a)

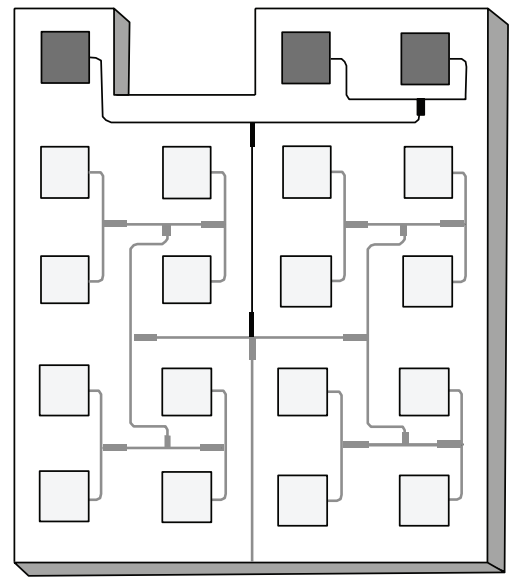

Irregular design 1

(array 3)

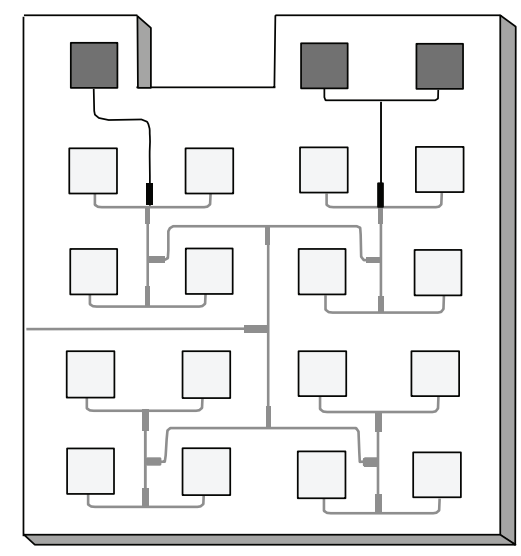

Irregular design 2 (array 2)

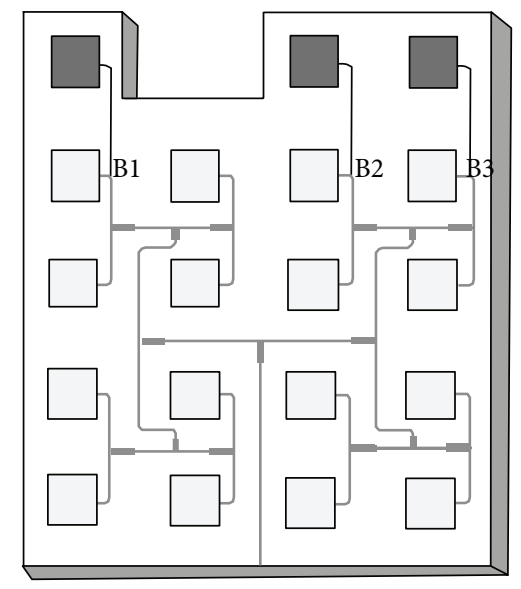

Irregular design 2 (array 4)

(b)

FIGURE 3: Designs of notched array (a) vertical and (b) horizontal polarization.

5 are distributed evenly which facilities the design of power dividers, where the power ratio in $\mathrm{C} 4$ is $3: 4: 4$.

Similar to the notched array, array 7 has a crowded feed line inside the basic sub-array unit. The strong coupling decreases the antenna aperture efficiency. Array 8 has a higher gain than array 7 , but at the lower frequency, its gain falls rapidly with frequency. This is because the modules $M_{1}$ and $M_{2}$ are serially fed from the basic unit. Nevertheless, it performs much better than the combined case with $M_{3}$.

Based on the simulated results, the array performances are compared in Table 1, where the total antenna efficiency is given by

$$
\eta_{\text {eff }}=\frac{G_{\text {simulated }}}{G_{\max }} .
$$

$G_{\text {simulated }}$ is the simulated gain and $G_{\max }=4 \pi A / \lambda^{2}$ is the maximum gain for a uniform aperture with the same aperture area A [16]. All irregular design arrays, containing 19 elements excited, have a gain of $0.5 \sim 0.7 \mathrm{~dB}$ higher than that of regular design array, which has only 16 elements excited, and hence the aperture efficiency is improved from $74 \%$ to $83 \sim 88.8 \%$. The loss due to the feed line is determined by the length and the reflection coefficient of junctions. Based on the simulations the used substrate causes about $0.155 \mathrm{~dB}$ power loss per wavelength. The feed loss for each sub-array is estimated and the results have been compared. It is known that the differences of aperture efficiency mainly come from the aperture field distribution. For the regular design, the low aperture efficiency is mainly due to the three special elements that are not used although the remaining 16 elements are uniformly excited. For the irregular design, the array 2, array 4 , and array 8 designs have a relatively high efficiency. The reason for this improvement was explained above. Not only the resulting array designs have a good performance, but also, as can be seen from the Figures, they have a very compact 


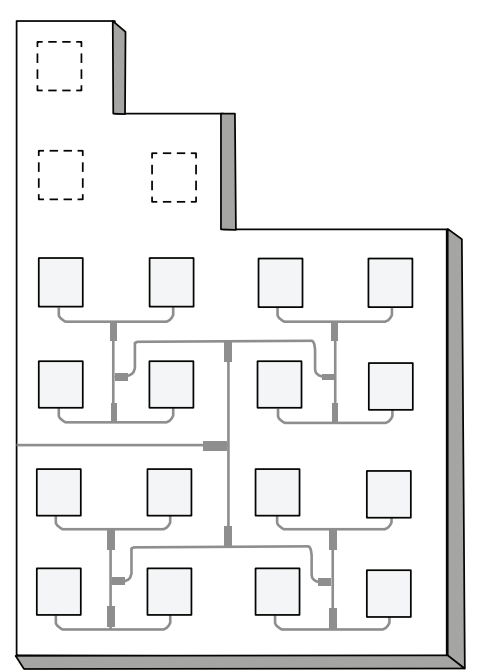

Regular design

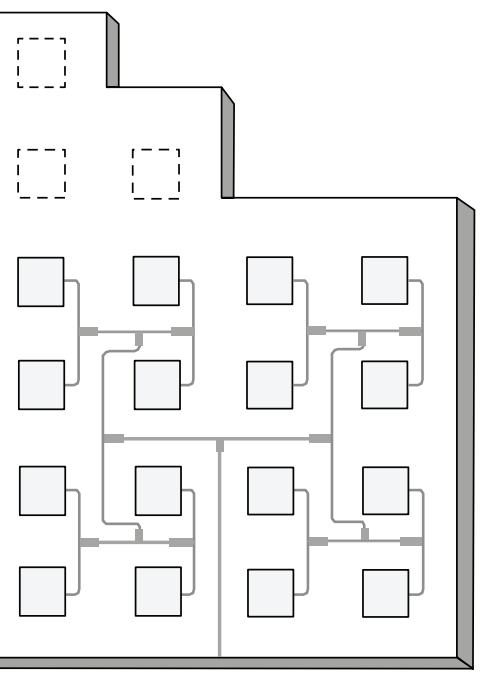

Regular design

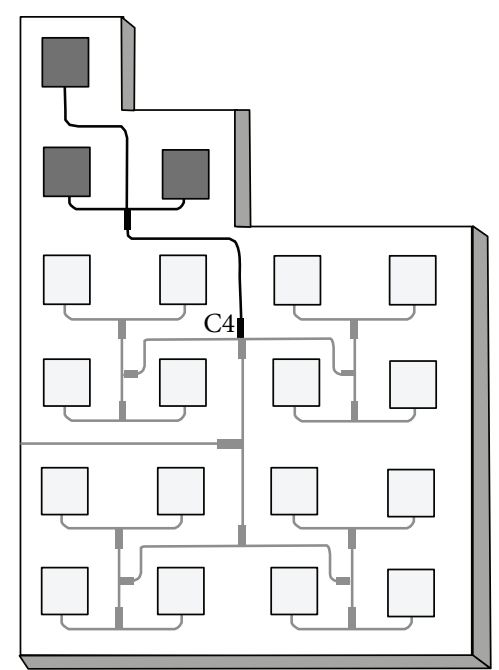

Irregular design 1 (array 5)

(a)

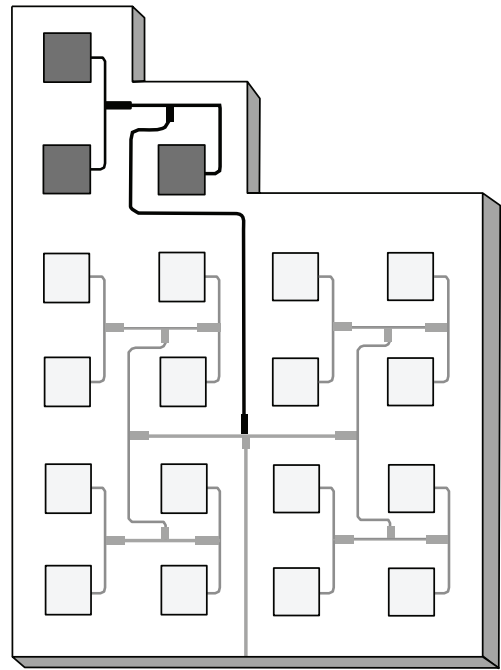

Irregular design 1

(array 7)

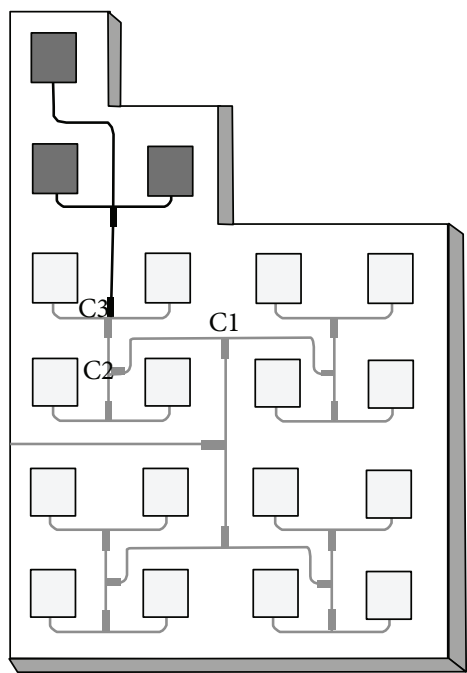

Irregular design 2 (array 6)

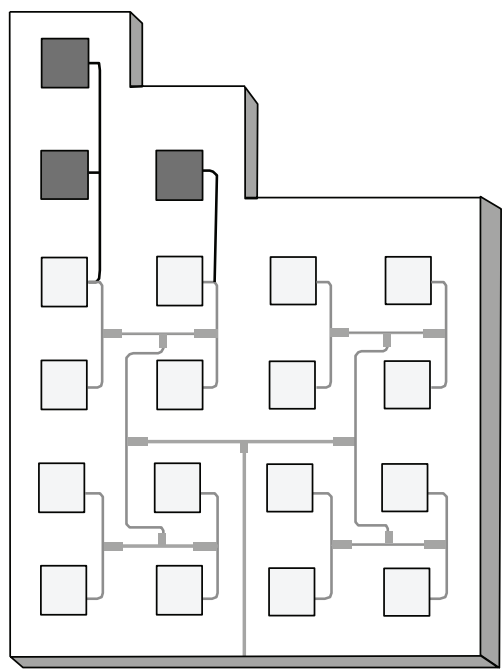

Irregular design 2 (array 8)

(b)

FIGURE 4: Designs of concave array (a) vertical and (b) horizontal polarization.

TABLE 1: Computed results of the subarrays.

\begin{tabular}{|c|c|c|c|c|c|c|}
\hline Design & Gain (dBi) & S.L.L (dB) & Cross-Pol. (dB) & Loss with feed line $(\mathrm{dB})$ & Aperture eff. & $\eta_{\text {eff }}$ \\
\hline Regular design & 19.30 & $\leq-13.4$ & $\leq-45$ & $\sim-0.69$ & $74 \%$ & $63.1 \%$ \\
\hline Array 1 & 19.90 & $\leq-13.3$ & $\leq-38$ & $\sim-0.70$ & $85.4 \%$ & $72.7 \%$ \\
\hline Array 2 & 20.05 & $\leq-13.4$ & $\leq-38$ & $\sim-0.70$ & $88.4 \%$ & $75.3 \%$ \\
\hline Array 3 & 19.80 & $\leq-13.3$ & $\leq-39$ & $\sim-0.70$ & $83.5 \%$ & $71.2 \%$ \\
\hline Array 4 & 20.05 & $\leq-13.4$ & $\leq-43$ & $\sim-0.71$ & $88.8 \%$ & $75.4 \%$ \\
\hline Array 5 & 19.90 & $\leq-13.5$ & $\leq-38$ & $\sim-0.70$ & $85.5 \%$ & $72.7 \%$ \\
\hline Array 6 & 19.75 & $\leq-13.6$ & $\leq-42$ & $\sim-0.72$ & $83.0 \%$ & $70.8 \%$ \\
\hline Array 7 & 19.85 & $\leq-13.3$ & $\leq-38$ & $\sim-0.71$ & $84.9 \%$ & $71.9 \%$ \\
\hline Array 8 & 19.95 & $\leq-13.5$ & $\leq-39$ & $\sim-0.72$ & $86.8 \%$ & $73.6 \%$ \\
\hline
\end{tabular}




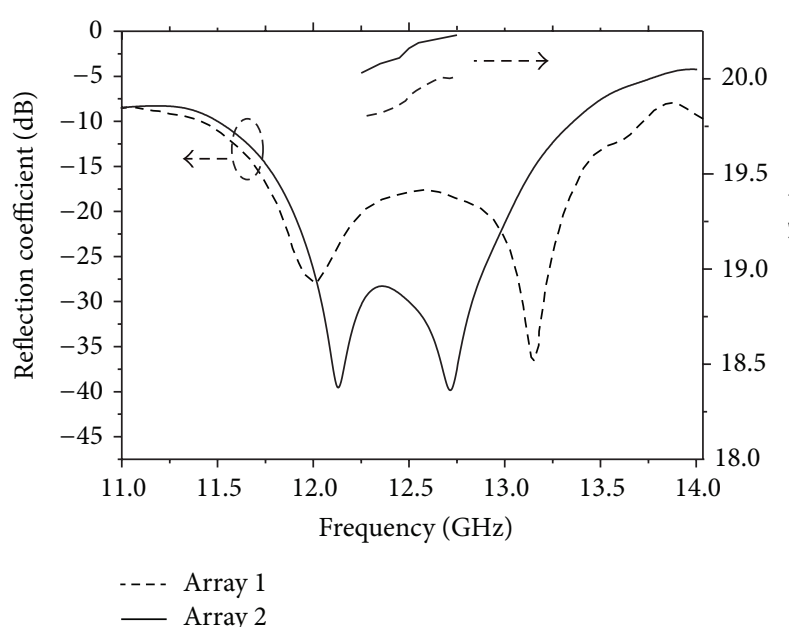

(a)

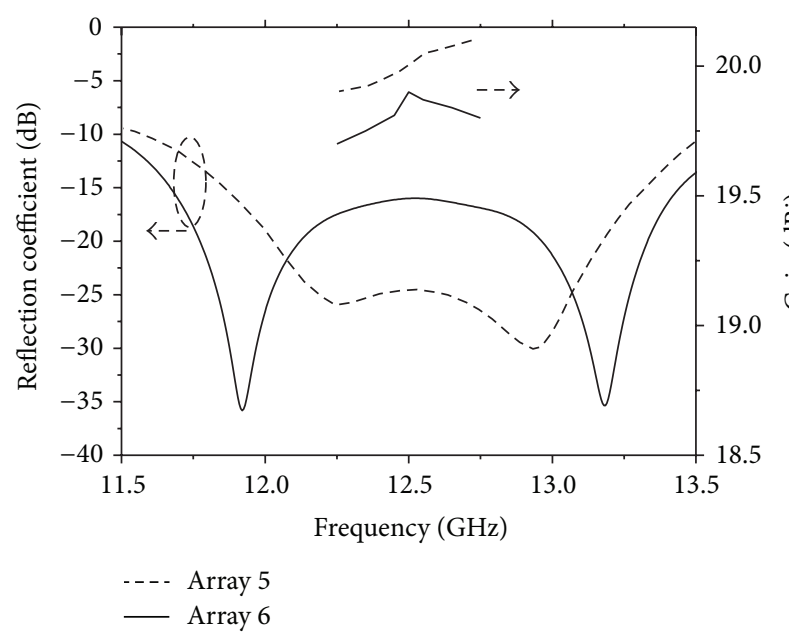

(c)

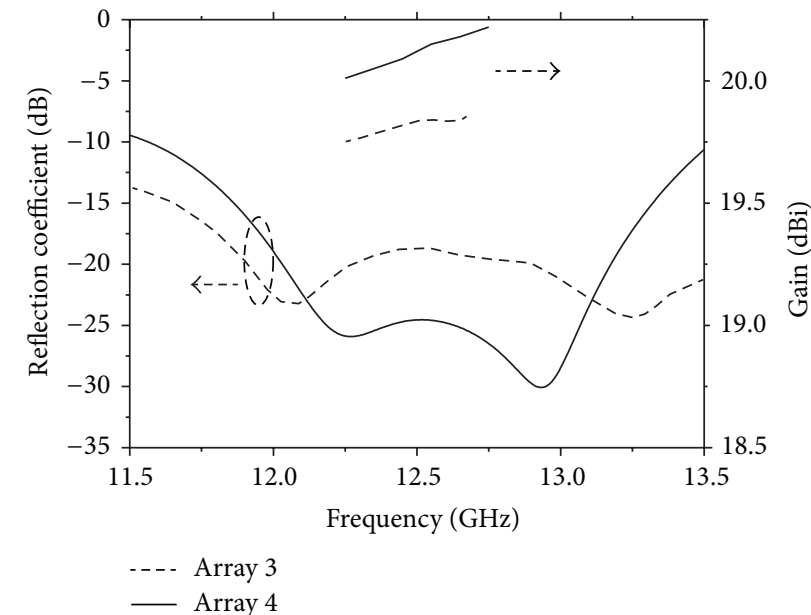

(b)

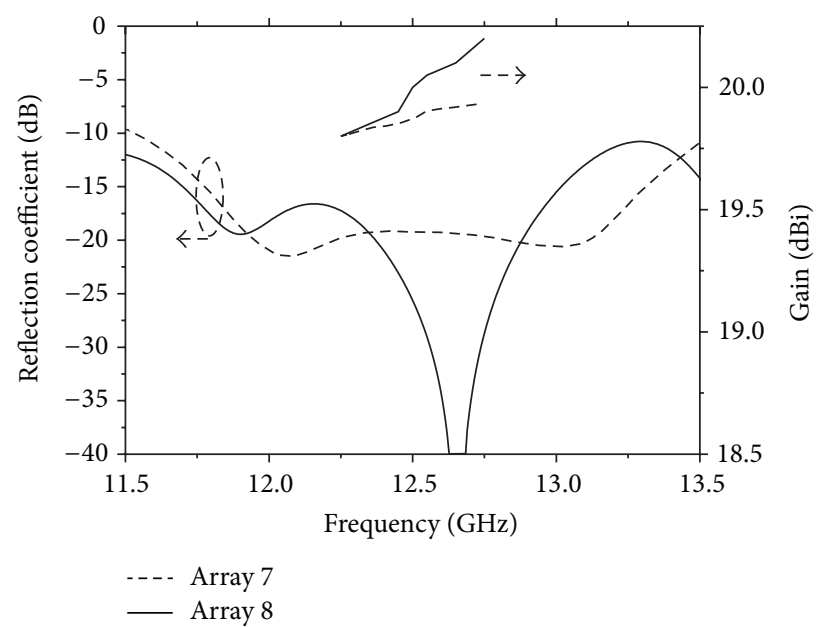

(d)

FiguRE 5: The simulated reflection coefficient and gain of the notched array (a) V-polarization, (b) H-polarization and concave array, (c) V-polarization, and (d) H-polarization.

layout, which is not constrained by the irregular shape of the array.

3.3. Design Guideline. From the illustration of the design and following discussion, it has been observed that all the irregular designs which exhibit a good performance have one trait in common; namely, the fewer changes to the main part of the feed network in the regular subaperture the better performance. In addition, the irregular subaperture should be considered an extension of the regular subaperture. This is reasonable because the regular subaperture plays the key role in radiation. Therefore, a general design guideline is that the layout must guarantee the performance of the regular subaperture, and then the potential of the irregular subaperture should be fully exploited without affecting the regular subaperture significantly.

A practical guideline can be described as follows: first, the arbitrarily shaped array is divided into two parts: a regular subaperture and an irregular one. The regular subaperture is designed using a symmetry technique. Then, according to the element distribution, the irregular subaperture is divided into several modules. Smaller modules are better. However, small modules often require a complicated feed network, so a tradeoff is needed to reach the highest antenna gain. The feed line to the modules is connected to the lower level junctions in basic unit of the regular subaperture to reduce unwanted coupling. The power distribution can be realized over a modest bandwidth ( 20\%) with a quarter wavelength transformer, which is adjusted as follows: the magnitude of current to the irregular modules is slightly increased or decreased until the gain of the array reaches its maximum value.

\section{Practical Array Design}

The design technique outlined above was applied to obtain a circular antenna array design over the bandwidth of 12.25$12.75 \mathrm{GHz}$. The diameter of the array is $325 \mathrm{~mm}$. The design 


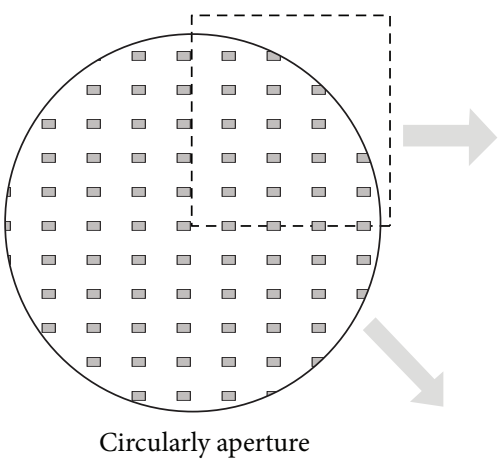

(a)

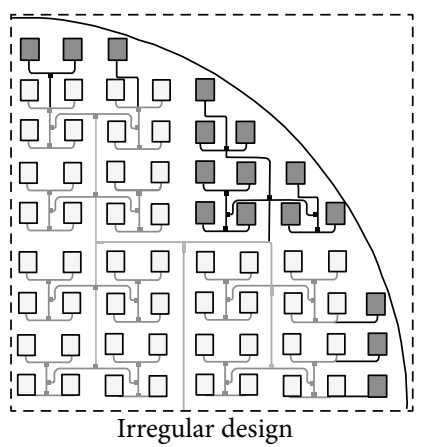

(c)

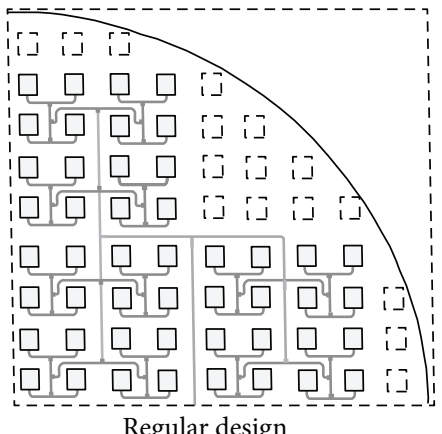

(b)

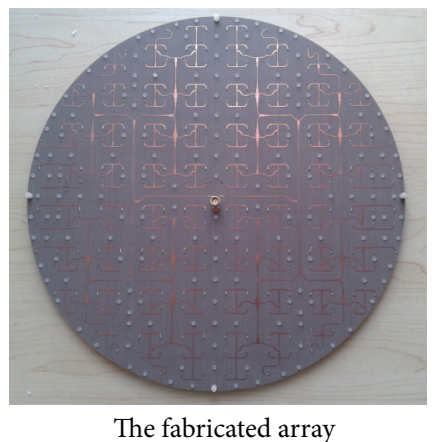

(d)

Figure 6: The layout of the circular array.

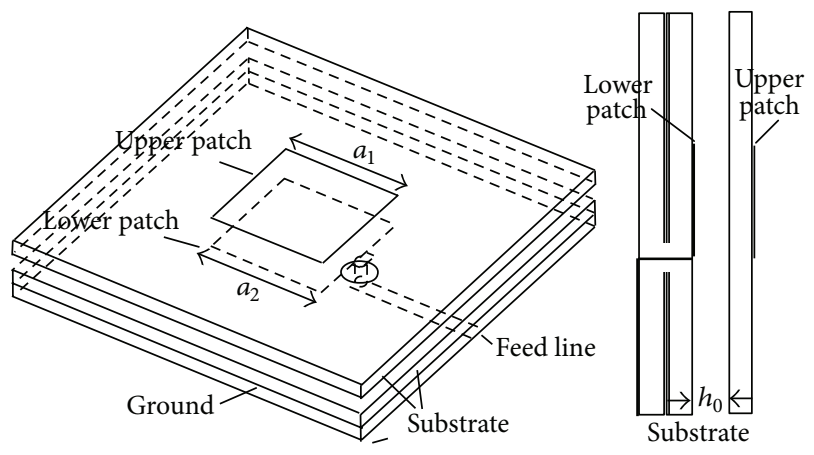

FIGURE 7: The array element design.

layout is illustrated in Figure 6. The aperture quadrant is divided into regular and irregular parts as shown. The regular subaperture was first filled based on a combination of parallel and serial feeding techniques to reduce the length of the feed line. The irregular subaperture was then composed of three modules: two $M_{3}$ and one $M_{10}$, and is further divided as follows: $M_{3}=3 \times M_{1}, M_{3}=M_{2}+M_{1}$, and $M_{10}=$ $M_{4}+M_{3}+M_{3}$; the small modules such as $M_{1}$ and $M_{2}$ are individually connected to the regular subaperture, while the modules separated from module $M_{10}$ are connected to each other since the $M_{10}$ can be integrated with the $4 \times 4$-element array of the regular subaperture directly.

For simplicity, a stacked patch antenna was selected for the antenna element [17]. Figure 7 shows the configuration

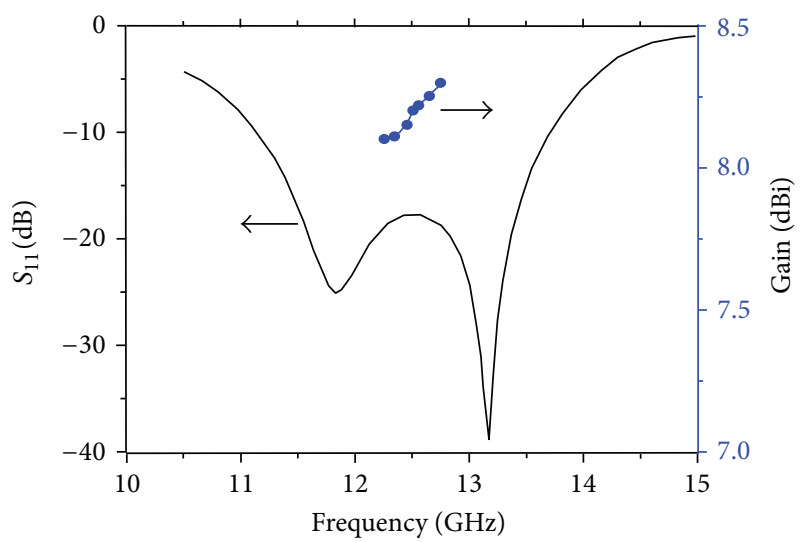

FIgURE 8: The reflection coefficient and gain of the element.

of the element, which consists of three substrate layers. All substrates have the same dielectric constant of $\varepsilon_{r}=2.55$ and thickness $h$. The upper square patch with a side length of $a_{1}$ is printed on the upper substrate while the lower square patch with a side length of $a_{2}$ is on the middle substrate. The distance between these two substrates is $h_{0}$. The microstrip feed network is printed on the bottom side of the substrate and is isolated from the patches through a metal ground plane, which frees up the design of feed network. The final optimized geometry of the antenna element for the frequency band of $12.25 \sim 12.75 \mathrm{GHz}$ is listed in Table 2. The substrate 


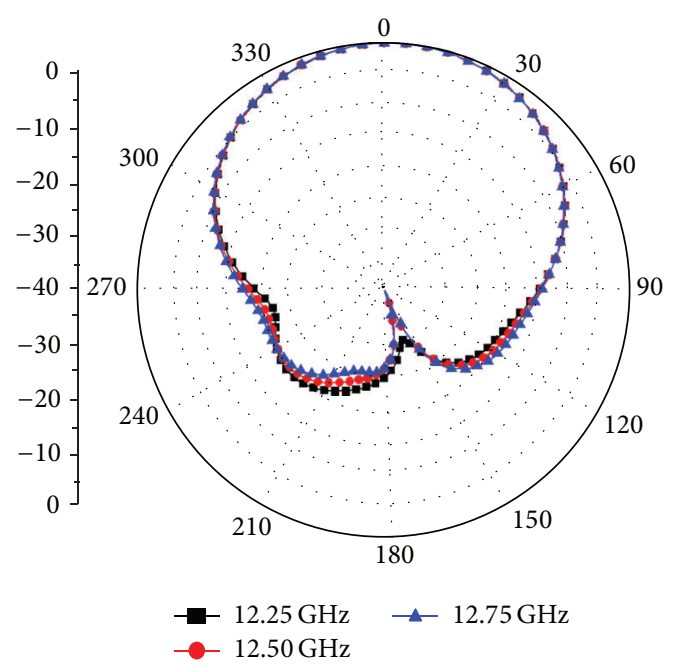

(a)

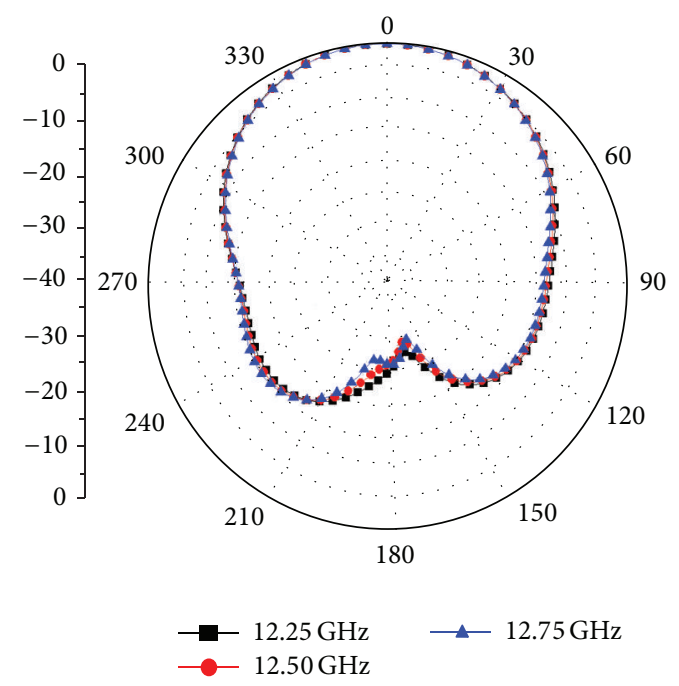

(b)

Figure 9: The normalized farfield patterns of the element at (a) E plane and (b) H plane.

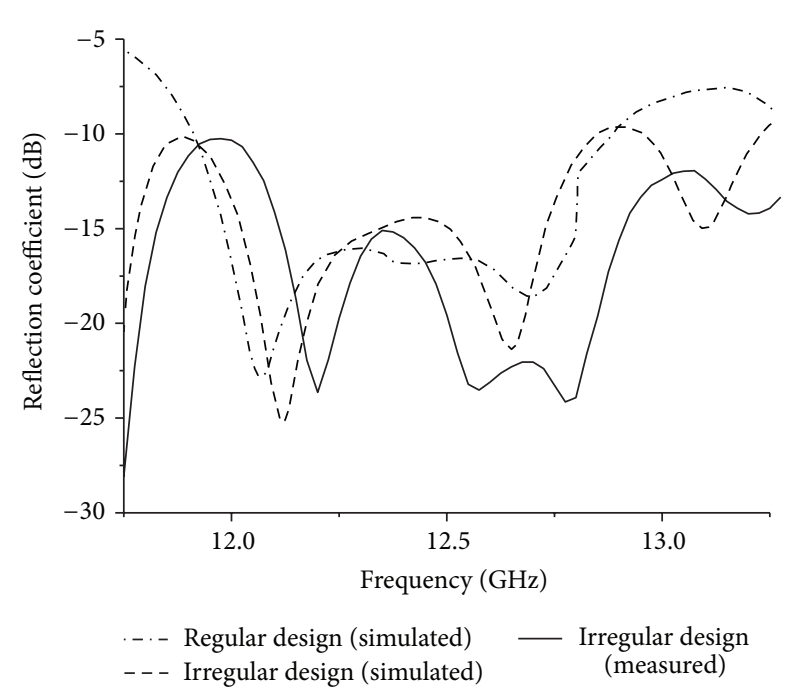

(a)

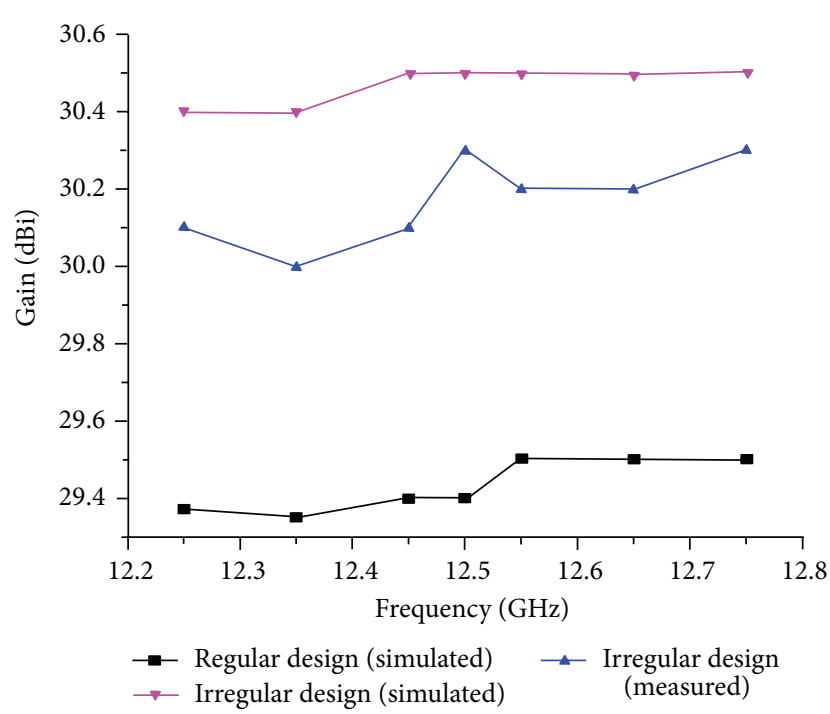

(b)

FIGURE 10: The (a) input reflection coefficient and (b) gain of the circular array.

TABLE 2: The parametric dimensions of the element $(\mathrm{mm})$.

\begin{tabular}{llll}
\hline$h$ & $h_{0}$ & $a_{1}$ & $a_{2}$ \\
\hline 0.762 & 1.3 & 3.5 & 3.5 \\
\hline
\end{tabular}

material used is from Arlon AD255. It has a loss tangent of 0.0018 at $10 \mathrm{GHz}$, and the conductor thickness is $0.035 \mathrm{~mm}$.

The simulated reflection coefficient of the antenna element is better than $-15 \mathrm{~dB}$ across the band as shown in Figure 8. The far-field patterns are plotted in Figure 9, and the half power beam widths are $66^{\circ}$ and $73.5^{\circ}$ for $\mathrm{E}$ and $\mathrm{H}$ planes, respectively. The antenna element gain is $8.1 \sim 8.3 \mathrm{dBi}$.
The antenna array spacing is $18 \mathrm{~mm}\left(0.75 \lambda_{0}\right.$, where $\lambda_{0}$ is the wavelength in free space at $12.5 \mathrm{GHz}$ ). Mutual coupling between elements is low at this distance, usually better than $-20 \mathrm{~dB}$. Figure 10 (a) shows the simulated reflection coefficient of the arrays. All the reflection levels are below $-15 \mathrm{~dB}$ in the operational band. The simulated gains are shown in Figure 10(b).

It is seen that the array with the regular design, employing only 192 of the elements, has a gain about $29.4 \mathrm{dBi}$. The full array with the irregular sub-array that uses 256 elements has a gain about $30.5 \mathrm{dBi}$. The maximum gain of the circular array is about $32.6 \mathrm{dBi}$ according to formula (1). As discussed above, the antenna efficiency is mainly limited by two aspects: the power loss of the feed network and the nonuniform aperture 


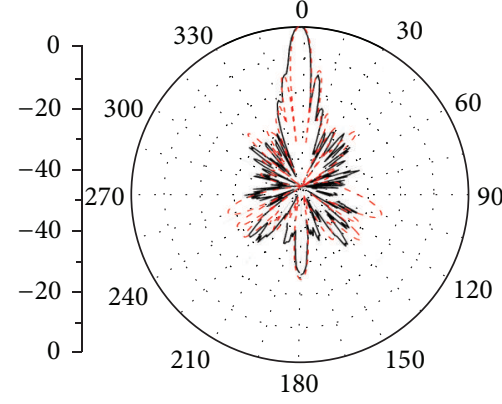

(a)

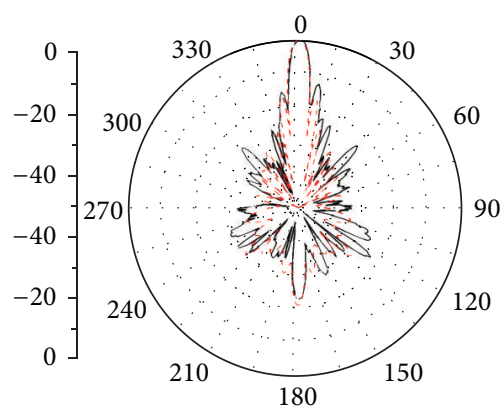

- Measured

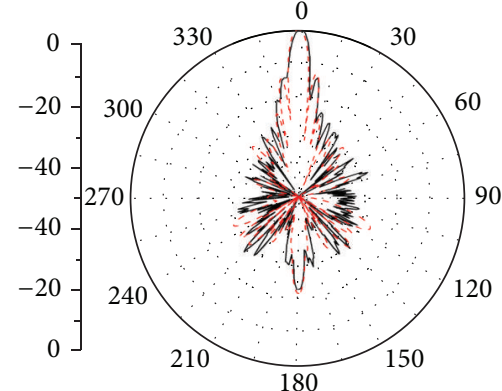

(b)

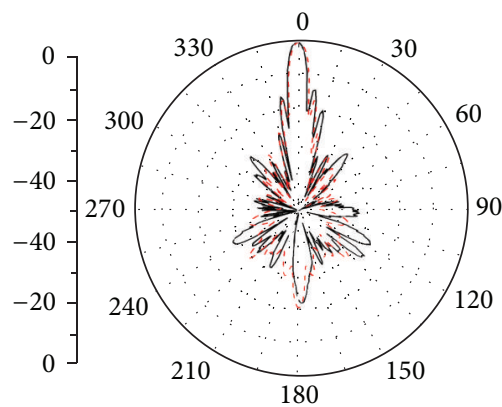

—- Measured
-- Simulated

(e)

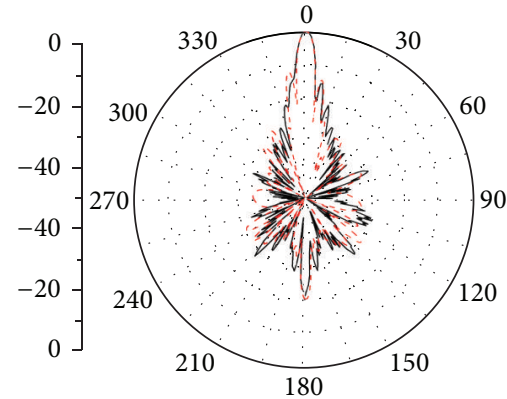

(c)

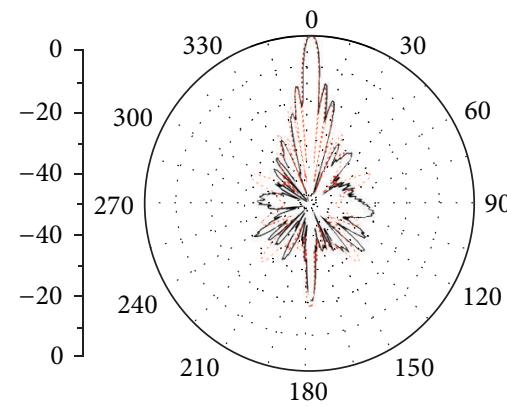

- Measured

(f)

Figure 11: The measured far-field patterns at (a) $12.25 \mathrm{GHz}, \mathrm{E}$ plane (b) $12.5 \mathrm{GHz}, \mathrm{E}$ plane (c) $12.75 \mathrm{GHz}, \mathrm{E}$ plane (d) $12.25 \mathrm{GHz}, \mathrm{H}$ plane (e) $12.5 \mathrm{GHz}, \mathrm{H}$ plane (f) $12.75 \mathrm{GHz}, \mathrm{H}$ plane.

field distribution. The power loss of the whole feed network with parallel and serial techniques is estimated to be about $1.7 \mathrm{~dB}$, and therefore the aperture efficiency is about $90 \%$.

As can be seen, the irregular subaperture is divided into many small modules, and the topology of feed network is compact. It is noted that the contribution of the feed network is not only that it has a short feed line, but also it has mostly protected the feed network of the regular subaperture.

To verify the design approach and the calculated results, a circular array based on the irregular design was fabricated. The resulting structure is shown in Figure 6. The reflection coefficient was measured with an Agilent 8722ES vector network analyzer (VNA) and the results are also shown in Figure 10(a). Good agreement is observed between the computed and measured results. The slight discrepancy may be due to the imperfect fabrication and cable connections.

The radiation patterns of the experimental circular array are shown in Figure 11 at various frequencies across the band. It can be seen that the simulated and measured results are in good agreement, except that the measured side lobes at the low frequency (Figure 11(d)) are slightly higher than those predicted, and the sidelobes in the $\mathrm{E}$ plane tend to merge. Since the feed network is isolated by the ground, the merging may be due to imperfections in the feed network as well as imperfect antenna alignment in the measuring process. All the side lobes are $13.2 \mathrm{~dB}$ or more below the peak level. The array gain was also measured by comparison with a standard horn antenna. The results obtained are plotted in Figure 10(b) and it can be seen that the measured gain agrees well with the computed result. The variation between the two is thought due to fabrication tolerances, ohmic loss, and assembly tolerances. A maximum gain of $30.3 \mathrm{dBi}$ was achieved at $12.5 \mathrm{GHz}$. Table 3 summarizes the radiation performance over the band where antenna efficiency is given by (1), except that $G_{\text {simulated }}$ is replaced by $G_{\text {measured }}$, the measured gain of the test array. The irregular design aperture efficiency is obviously higher than the regular design, and it causes very little cross-polarization component in the band.

\section{Conclusion}

A design technique for a high efficiency arbitrarily shaped planar microstrip antenna array is described. In this design technique, the shape of the array is treated as a combination of regular and irregular subapertures. For the regular subaperture, a conventional symmetry technique is used to obtain a high performance, and for the irregular subaperture, small modules are used to construct the array. The selection of modules as well as the topology of feed network is optimized to increase the antenna gain. Based on this decomposition of the array aperture, a circular aperture array was designed and fabricated to demonstrate the approach. The agreement between the computed and measured results verified the effectiveness of the design technique. The design approach for 
TABle 3: The performance of the circular array.

\begin{tabular}{|c|c|c|c|c|c|c|c|c|c|c|c|}
\hline \multirow{3}{*}{$\begin{array}{l}\text { Frequency } \\
(\mathrm{GHz})\end{array}$} & \multicolumn{8}{|c|}{ Simulated } & \multirow{2}{*}{\multicolumn{3}{|c|}{$\begin{array}{c}\text { Measured } \\
\text { Irregular deign }\end{array}$}} \\
\hline & \multicolumn{4}{|c|}{ Regular design } & \multicolumn{4}{|c|}{ Irregular design } & & & \\
\hline & Gain $(\mathrm{dBi})$ & $\eta_{\text {eff }}$ & $X$-Pol. (dB) & S.L.L (dB) & Gain (dBi) & $\eta_{\mathrm{eff}}$ & $X$-Pol. (dB) & S.L.L (dB) & Gain (dBi) & $\eta_{\mathrm{eff}}$ & S.L.L. (dB) \\
\hline 12.25 & 29.3 & $49.7 \%$ & $\leq-48$ & $\leq-13.7$ & 30.4 & $63.0 \%$ & $\leq-45$ & $\leq-13.7$ & 30.1 & $58.8 \%$ & $\leq-13.2$ \\
\hline 12.50 & 29.4 & $48.1 \%$ & $\leq-48$ & $\leq-13.9$ & 30.5 & $61.9 \%$ & $\leq-43$ & $\leq-14.2$ & 30.3 & $59.2 \%$ & $\leq-14.1$ \\
\hline 12.75 & 29.5 & $47.3 \%$ & $\leq-44$ & $\leq-13.7$ & 30.5 & $59.5 \%$ & $\leq-40$ & $\leq-13.6$ & 30.3 & $55.3 \%$ & $\leq-13.8$ \\
\hline
\end{tabular}

arrays ensures better utilization of the limited system space available. In addition, the design technique is simple and easily realized with planar circuit fabrication, making it very suitable to various wireless communications applications.

\section{Acknowledgments}

This work was supported by the National Science and Technology of Major Project (2011ZX03001-007-03), the National Natural Science Foundation (61201058), the Research and Innovation Project of Shanghai Education Commission (12Z112030001), and the Scientific Research Foundation for Returned Overseas Chinese Scholars, State Education Ministry, the Project of "SMC Excellent Young Faculty."

\section{References}

[1] Y.-B. Jung, S.-Y. Eom, and S.-I. Jeon, "Novel antenna system design for satellite mobile multimedia service," IEEE Transactions on Vehicular Technology, vol. 59, no. 9, pp. 4237-4247, 2010.

[2] S. Y. Eom, S. H. Son, Y. B. Jung et al., "Design and test of a mobile antenna system with tri-band operation for broadband satellite communications and DBS reception," IEEE Transactions on Antennas and Propagation, vol. 55, no. 11, pp. 3123-3133, 2007.

[3] J. M. Fernández González, P. Padilla, G. Expósito-Domínguez, and M. Sierra-Castañer, "Lightweight portable planar slot array antenna for satellite communications in X-band," IEEE Antennas and Wireless Propagation Letters, vol. 10, pp. 1409-1412, 2011.

[4] J. Hirokawa, M. Ando, N. Goto, N. Takahashi, T. Ojima, and M. Uematsu, "A single-layer slotted leaky waveguide array antenna for mobile reception of direct broadcast from satellite," IEEE Transactions on Vehicular Technology, vol. 44, no. 4, pp. 749755, 1995.

[5] Y. Hase, N. Obara, H. Saitoh, and C. Ohuchi, "Slot array antenna system for COMETS advanced mobile satcom experiments," in Proceedings of the IEEE 46th Vehicular Technology Conference (VTC '96), vol. 1, pp. 353-356, Atlanta, Ga, USA, May 1996.

[6] S. Vaccaro, F. Tiezzi, M. F. Rúa, and C. D. G. De Oro, "Ku-band low-profile Rx-only and Tx-Rx antennas for mobile satellite communications," in Proceedings of the IEEE International Symposium on Phased Array Systems and Technology, pp. 536542, Waltham, Mass, USA, October 2010.

[7] J. Wang and J. H. Winters, "An embedded antenna for mobile DBS," in Proccedings of the IEEE 60th Vehicular Technology Conference (VTC '04), vol. 6, pp. 4092-4095, Los Angeles, Calif, USA, September 2004.

[8] M. W. Shelley and J. Vazquez, "Low profile scanning antennas for sitcom 'on-the-move,"' in IEEE Military Communications conference, vol. 2, pp. 705-709, 1993.
[9] E. Levine, G. Malamud, S. Shtrikman, and D. Treves, "A study of microstrip array antennas with the feed network," IEEE Transactions on Antennas and Propagation, vol. 37, no. 4, pp. 426-434, 1989.

[10] R. Azadegan, "A Ku-band planar antenna array for mobile satellite tv reception with linear polarization," IEEE Transactions on Antennas and Propagation, vol. 58, no. 6, pp. 2097-2101, 2010.

[11] M. E. Bialkowski and N. C. Karmakar, "A two-ring circular phased-array antenna for mobile satellite communications," IEEE Antennas and Propagation Magazine, vol. 41, no. 3, pp. 1423, 1999.

[12] Y. Liu, L.-M. Si, M. Wei et al., "Some recent developments of microstrip antenna," International Journal of Antennas and Propagation, vol. 2012, Article ID 428284, 10 pages, 2012.

[13] Raysat Cyprus Limited, "Flat microwave antenna," World Intellectual Property Organization, WO2005/004284 A1.

[14] H. D. Chen, C.-Y.-D. Sim, J.-Y. Wu, and T.-W. Chiu, "Broadband high-gain microstrip array antennas for WiMAX base station," IEEE Transactions on Antennas and Propagation, vol. 60, no. 8, pp. 3977-3980, 2012.

[15] N. Herscovici, "New considerations in the design of micro strip antennas," IEEE Transactions on Antennas and Propagation, vol. 46, no. 6, pp. 807-812, 1998.

[16] C. L. Lin and Z. P. Nie, Antenna Engineering Handbook, Publishing House of Electronics Industry, Beijing, China, 2002.

[17] Z.-N. Chen, X.-L. Liang, S. Ye et al., "A novel dual-polarization microstrip antenna array with wideband and high gain in $\mathrm{Ku}$ band," Chinese Journal of Radio Science, vol. 26, no. 4, pp. 661665, 2011. 

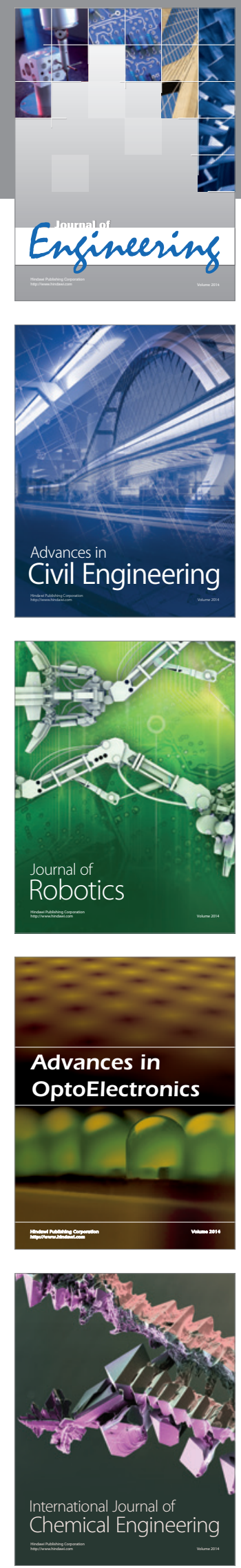

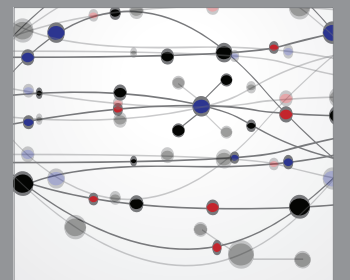

The Scientific World Journal
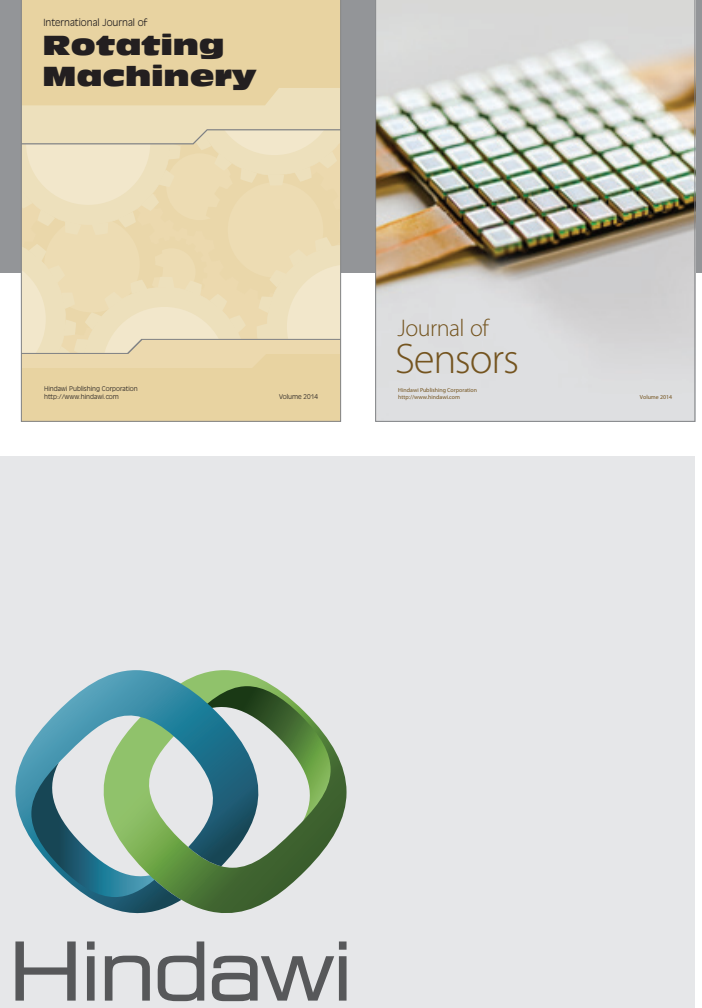

Submit your manuscripts at http://www.hindawi.com
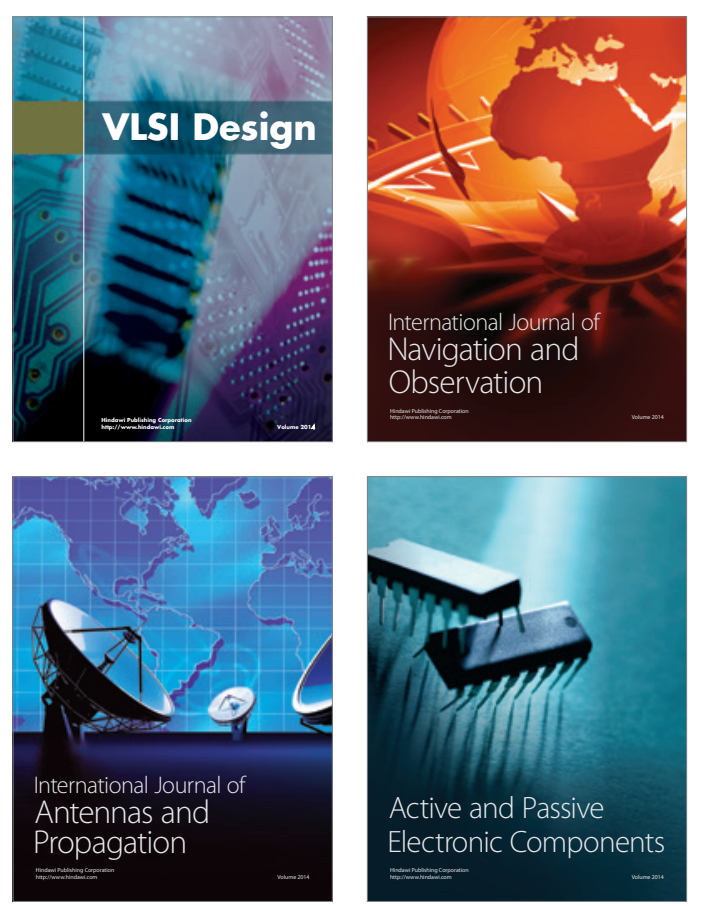
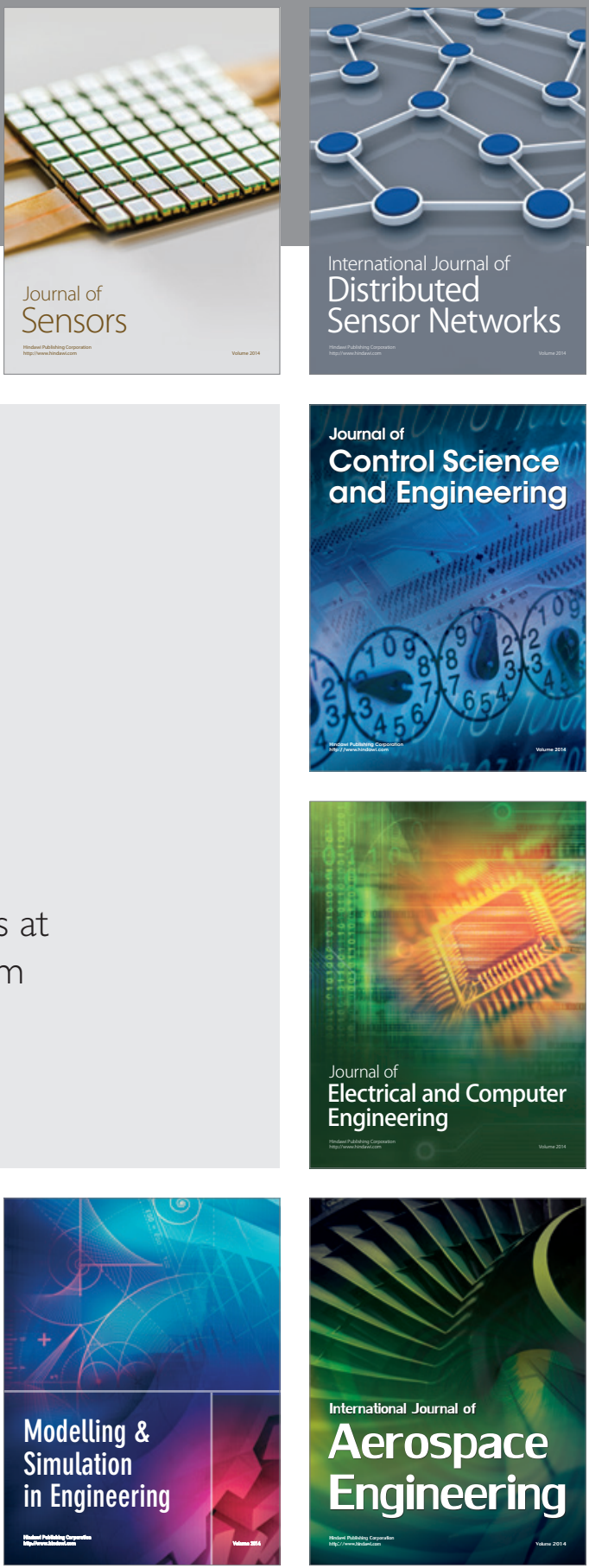

Journal of

Control Science

and Engineering
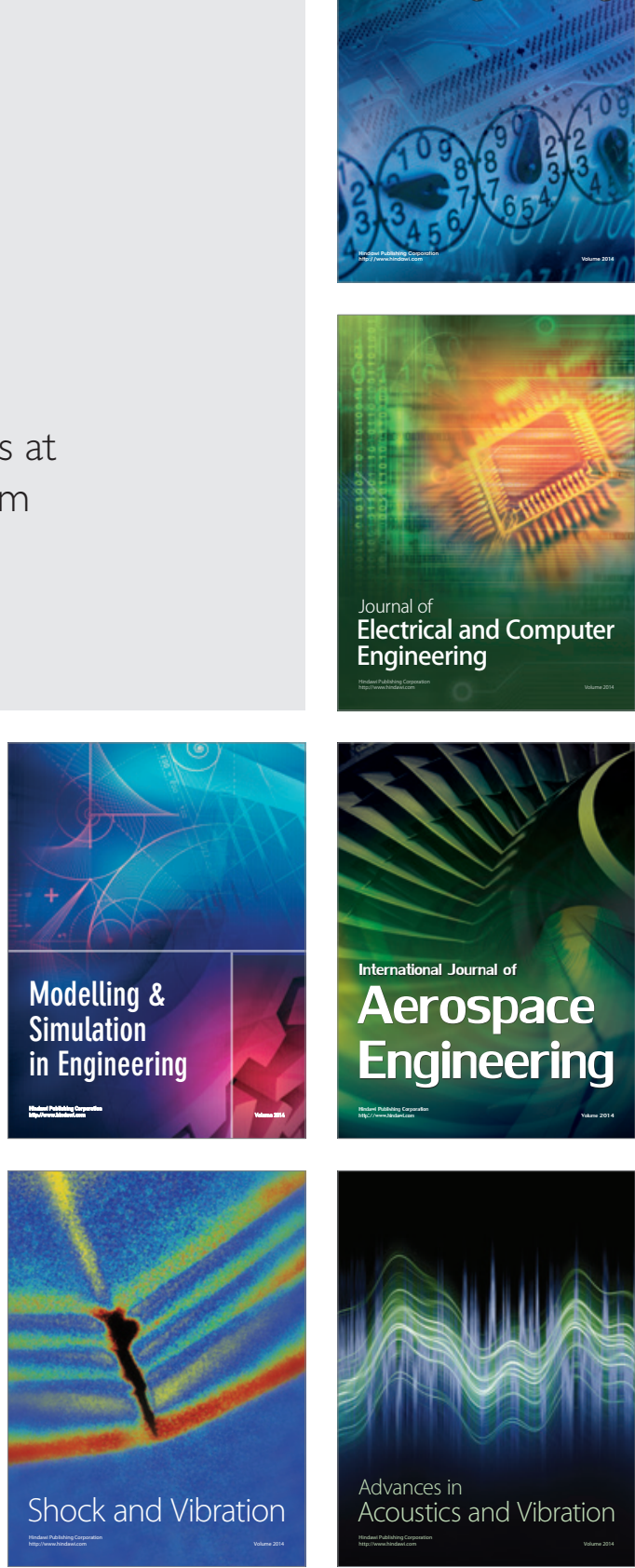